\title{
Anti-inflammatory and Anti-thrombotic Efficacy of Targeted Ultrasound Microbubbles on LPS-induced HUVEC Cells
}

\author{
JINDONG SUN ${ }^{1}$, SHIJUN PAN ${ }^{2}$, HUAMIN YU ${ }^{1}$, HAIQIANG HU ${ }^{1}$, YU SUN ${ }^{3}$, \\ ZHIJIAN YANG ${ }^{4}$, ROBERT M. HOFFMAN ${ }^{4}$ and HONG YUAN ${ }^{1}$ \\ ${ }^{1}$ First People's Hospital of Linping District, Hangzhou, P.R. China; \\ ${ }^{2}$ Hangzhou Normal University, Hangzhou, P.R. China; \\ ${ }^{3}$ Department of Surgery, UCSD, San Diego, CA, U.S.A; \\ ${ }^{4}$ AntiCancer Inc., San Diego, CA, U.S.A.
}

\begin{abstract}
Background/Aim: The early stage of atherosclerosis (AS) demonstrates a lipid-driven inflammatory cytokine increase. In the present study, we aimed to use ultrasoundtargeted microbubble delivery (UTMD) therapy with the Endostar-loaded target microbubbles $(M B S)$ to reduce $A S$ related inflammatory response. Materials and Methods: Normal and lipopolysaccharide (LPS) induced human umbilical vein endothelial cells (HUVECs) were placed in a parallel-plate flow chamber. MBs were perfused through the parallel-plate flow chamber to mimic physiological blood flow. Five groups were set up: G1: Negative control (normal HUVECS); G2: LPS control (LPS induced HUVECS); G3: ICAM-1-loaded-MBs (MBi); G4: Endostar-loaded-MBs (MBe) and G5: Endostar-ICAM-1-loaded-MBs (MBei). mRNA expression of inflammatory factors and release of inflammatory cytokines were detected by RT-PCR and ELISA, respectively. Results: After treatment with MBei, the mRNA expression of cell adhesion molecule-1 (CD31) $(p=0.004)$, endothelin-1 (ET1) $(p=0.010)$, von willebrand factor $(v W F)(p=0.018)$, extracellular regulated protein kinases $(E R K)(p=0.046)$ and nuclear factor kappa $B(N F-k B)(p=0.003)$ were significantly reduced compared to LPS-induced HUVECs. Release of inflammatory cytokines including tissue factor $(T F)(p=0.033)$, tissue factor pathway inhibitor (TF-PI) $(p=0.019)$, ET-1 $(p=0.014), v W F(p=0.030)$ and blood-coagulation factor VII $\alpha$ $(F V I I \alpha)(p=0.000)$ were also significantly reduced compared to LPS-induced HUVECs. Conclusion: UTMD therapy can
\end{abstract}

This article is freely accessible online.

Correspondence to: Hong Yuan, First People's Hospital of Linping District, Hangzhou, 311100, PR China. Tel: +86 057189369926 , e-mail: halloween0708@163.com

Key Words: Atherosclerosis, inflammatory cytokines, LPS-induced HUVECs, ultrasound microbubbles, parallel-plate flow chamber. inhibit the inflammatory response by reducing atheroscleroticrelated inflammatory factors, suggesting a potential treatment at the early-stage of $A S$.

Atherosclerosis (AS) is a lipid-driven inflammatory disease. Formation of atherosclerotic plaques is widely believed to be associated with hyperlipidemia, endothelial dysfunction, inflammation and neoangiogenesis in the plaque (1-5). In our previous study, we reported the inhibitory effect of an ultrasound-targeted microbubble delivery (UTMD) therapy in an atherosclerotic APOE-deficient mouse model. The drug-loaded microbubbles (MBs) targeted intraplaque neovascularization by conjugating microbubbles with an angiogenesis inhibitor Endostar and an antibody against ICAM-1 (intercellular adhesion molecule-1) and inhibited the formation of atherosclerotic plaque in vivo (6).

The parallel-plate flow chamber is a common platform to mimic blood vessel wall shear stress in vitro (7), which can be used to monitor the dynamics of flow through the microfluidic flow chamber in real time. In recent years, researchers have realized that wall shear stress is a key factor in mediating exercise-induced endothelial function variations (8). In the present study, we seeded the lipopolysaccharide (LPS) induced human primary umbilical vein endothelial cells (HUVECs) in a parallel-plate flow chamber (9) to mimic the physiological conditions of neovascularization and inflammation in the endothelial cells (ECs) at the early stage of AS in vivo. Endostar-loaded-MBs with targeting ICAM-1 antibody were used to treat LPS induced HUVECs by UTMD. Anti-inflammation efficacy was evaluated by detection of atherosclerotic-related inflammatory factors.

\section{Materials and Methods}

Ligand conjugation of microbubbles. One vial $(800 \mu \mathrm{L})$ of streptavidin-labeled ultrasound microbubbles (MBs) USphere ${ }^{\mathrm{TM}}$ Labeler-LS (TRUST Bio-sonics, Zhubei, Taiwan, ROC) was 

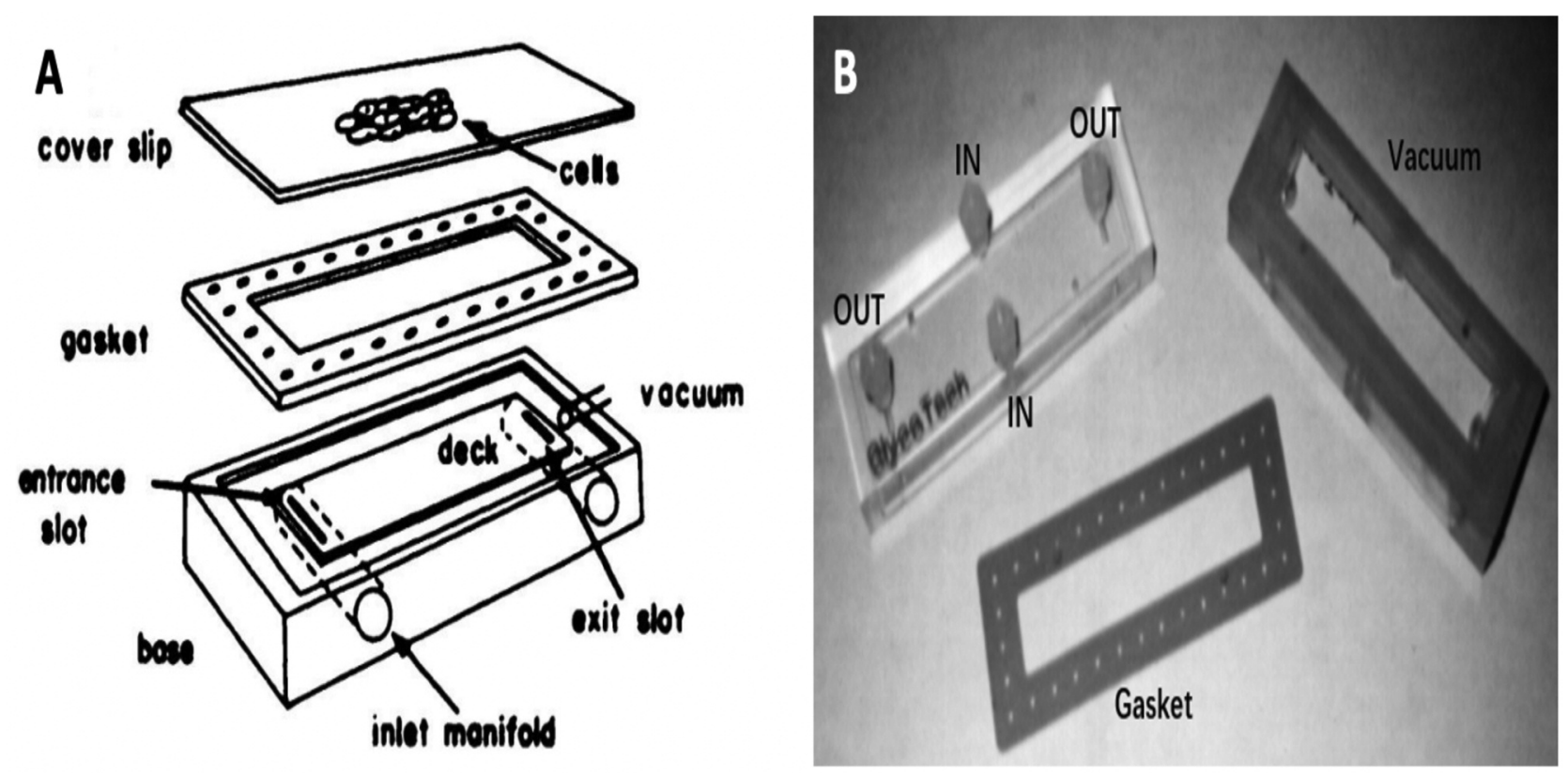

Figure 1. Presentation of the parallel-plate flow chamber. (A) Schematic diagram of the parallel-plate flow chamber; (B) Parallel-plate flow chamber equipment.

activated by agitation for 40-seconds using an agitator (TD-1001, UltraMix $^{\text {TM }}$, Zhubei, Taiwan, ROC). Endostar (Simcere Pharmaceutical, Nanjing, P.R.C.) was labeled with biotin and fluorophore rhodamine (Thermo Fisher, Waltham, MA, USA). ICAM-1 (Abcam, Cambridge, MA, USA) was labeled with biotin and fluorophore FICT (Thermo Fisher). FICT-biotin-labeled ICAM$1(50 \mu \mathrm{g})$ and rhodamine-biotin-labeled Endostar $(50 \mu \mathrm{g})$ were injected into the Labeler-LS vial and incubated at $4{ }^{\circ} \mathrm{C}$ for $30 \mathrm{~min}$ with gentle rotation to mix the solutions well. The incubated solution was centrifuged at $1,000 \times g$ for $3 \mathrm{~min}$. A syringe with a long needle was then used to remove the non-conjugated solution on the lower layer and replace it with fresh phosphate buffer saline (PBS). The ratio of MBs conjugated with both Endostar and ICAM-1 was determined using fluorescence microscopy and flow cytometry.

Cell culture and lipopolysaccharide (LPS) induction. Human umbilical vein endothelial cells (HUVECs) were obtained from ATCC (Manassas, VA, USA). Cells were cultured on slide plate and maintained in vascular-cell basal medium (ScienCell ${ }^{\mathrm{TM}}$, CAS \#: 1001, Carlsbad, CA, USA) supplemented with $10 \%$ heat-inactivated fetal- bovine serum (Lonsera Inc., CAS \#: S711-001S, Canelones, Uruguay) and 1\% streptomycin (NCM Biotech, CAS \#: C125C5, Suzhou, Jiangsu, PR China). The cells were incubated at $37^{\circ} \mathrm{C}$ with a $5 \% \mathrm{CO}_{2}$. The cells were treated with lipopolysaccharide (LPS) (Sigma, CAS \#: C201005, Louis, MO, USA) at $5 \mu \mathrm{g} / \mathrm{ml}$ for $4 \mathrm{~h}$.

Microbubble adhesion and cavitation therapy using a parallel-plate flow chamber. The parallel-plate flow chamber system (GlycoTech, Gaithersburg, MD, USA) consists of three parts as are described by Lawrence et al. (10) (Figure 1). The parallel-plate flow chamber contains (i) a base plate with an entrance and exit port through which cells and media are perfused, and a vacuum outlet so that the apparatus can be held in place; (ii) a plastic slide plate on which the substrate is placed and (iii) a gasket that controls the chamber diameter. The polycarbonate shear deck supports a glass slide held in place by a vacuum applied through holes in a silastic spacing gasket. Fluid flow occurs through a slotted manifold to maintain a fully uniform laminar flow over the entire length of the chamber (11).

The parallel-plate flow chamber was pre-warmed to $37^{\circ} \mathrm{C}$ before the flow assay. The slide plate was seeded with normal or LPS induced HUVECs and placed in the flow chamber. Naked-MBs, $\mathrm{MBe}, \mathrm{MBi}$ or MBei were injected through the inlet lane at a shear stress of 2.0 dynes $/ \mathrm{cm}^{2}$. The real-time perfusion of MBs was monitored by contrast-enhanced ultrasound imaging (CEUS) using a Vevo 2100 ultrasound scanner (VisualSonics, Toronto, ON, Canada) with a linear transducer. Microbubble adhesion to HUVECs was examined using a microscope (TE2000, Nikon, Tokyo, Japan), and the number of bonded microbubbles in each group was counted.

Ultrasound cavitation was performed using a sonicator machine with a $1 \mathrm{~cm}^{2}$ cavitation tip. The tip was placed towards the bottom of the parallel-plate flow chamber and coupled using an acoustic coupling gel. The parameter of cavitation was set up at a frequency of $238 \mathrm{kHz}, 500 \mathrm{mV}, 0.5 \mathrm{MPa} 60$-s sonication duration, 10 pulses with 10 -ms pulse length and 50\% duty cycle (Figure 2 ).

Five groups were set up for the flow assay. G1: Negative control (normal HUVECs); G2: LPS control; G3: ICAM-1-loaded-MBs (MBi); G4: Endostar-loaded-MBs (MBe); G5: Endostar-ICAM-1-loaded-MBs (MBei). The images before- and post-cavitation were captured and analyzed using Vevo 2100 ultrasound scanner and its software.

Reverse transcription-polymerase chain reaction (RT-PCR). Trizol (Invitrogen, Carlsbad, CA, USA) was used to isolate RNA from the HUVECs according to the manufacturer's protocol. The VazymE reverse transcription kit (R123-01, Vazyme Biotech, Nanjing, Jiangsu, 


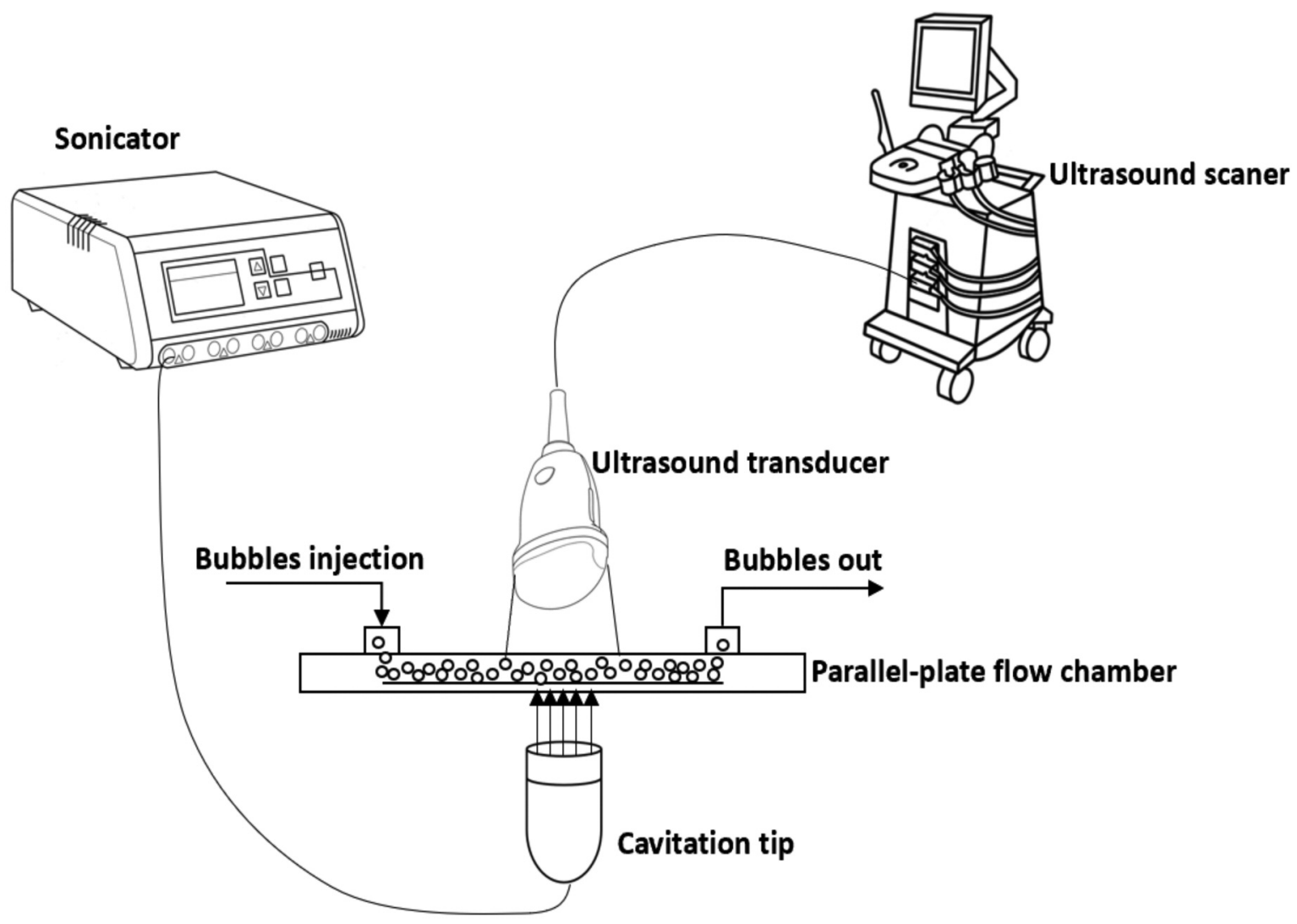

Figure 2. Schematic diagram of ultrasound monitoring and cavitation. An ultrasound cavitation is performed when the MBs flow through the parallelplate flow chamber with a sonicator. The images before and after cavitation are recorded with an ultrasound scanner.

PR China) was used to reversely transcribe RNA into cDNA. The following specific primers for each gene were used for PCR amplification: Platelet endothelial cell adhesion molecule-1 (CD31), endothelin-1 (ET-1), von Willebrand factor (vWF), extracellular regulated protein kinases (ERK) and protein of nuclear factor kappa $\mathrm{B}$ (NF-kB). Primer sequences are as following: CD31 (F: 5'GGCTTGTAGTTTTGGCTAGTCCTTG-3'; R: 5'-TGCTTGTTCCA CCTTCATTTTCTG-3'); ET-1 (F: 5'-AAGGTCCTAAGGGAGTGTT TGTGTC-3'; R: 5'-TTGCATGTTTGTCTTTTGAGTGTGT-3'); vWF (F: 5'-CCGATGCAGCCTTTTCGGA-3'; R: 5'-TCCCCAAGATA CACGGAGAGG-3'); ERK (F: 5'-GAAGGCCCGAAACTACCT ACAG-3'; R: 5'-TAAAGGTTAACATCCGGTCCAG-3'); NF-KB (F: 5'-TGTCCTTTCTCATCCCATCTTTG-3'; R: 5'-TGTCCTCTTTC TGCACCTTGTC-3'). Quantitative RT-PCR was carried out using the Step One Plus тм real-time PCR system [Applied Biosystems (Waltham, MA, USA)]. Relative RNA expression was calculated with the $2^{-\Delta \Delta \mathrm{Ct}}$ method.

Enzyme-linked immunosorbent assay (ELISA). The supernatant of each group was collected to detect and quantitate the release of inflammation-related cytokines including tissue factor (TF), tissue factor pathway inhibitor (TF-PI), ET-1, vWF and blood-coagulation factor VII $\alpha$ (FVII $\alpha)$, before and after ultrasound cavitation. Microtiter plates coated with the monoclonal antibodies were incubated with supernatants and washed, before the second enzyme-linked polyclonal antibodies were added. Following incubation, the plates were washed to remove unbound antibodies. A color substrate was added and allowed to react with horseradish peroxidase (HRP) for $20 \mathrm{~min}$. The reaction was stopped with $\mathrm{HCl}$. Absorbance was read at $450 \mathrm{~nm}$ to measure protein concentrations with a sensitivity of 12.5 or $25 \mathrm{pg} / \mathrm{ml}$. All treatments were performed in triplicate and each experiment was performed at least 3 times.

ELISA kits specification: TF (DCF300, R\&D Systems, Inc., Minneapolis, MN, USA); TF-PI (DTFP10, R\&D Systems, Inc.); vWF (DY2764-05, R\&D Systems, Inc.); ET-1 (ab158332, Abcam, Cambridge, MA, USA); FVII $\alpha$ (ab272771, Abcam).

Statistical analysis. All statistical analyses were performed using GraphPad Prism 8.0 (GraphPad Software, Inc. La Jolla, CA, USA). Significant differences between groups were calculated using oneway variance (ANOVA) followed by the Tukey correction. The data are expressed as the mean \pm standard deviation (SD). A $p$-value of $<0.05$ was considered to indicate statistical significance.

\section{Results}

Microbubble conjugation. Microbubbles were conjugated with ICAM-1 antibody and analyzed by epifluorescence microscopy 

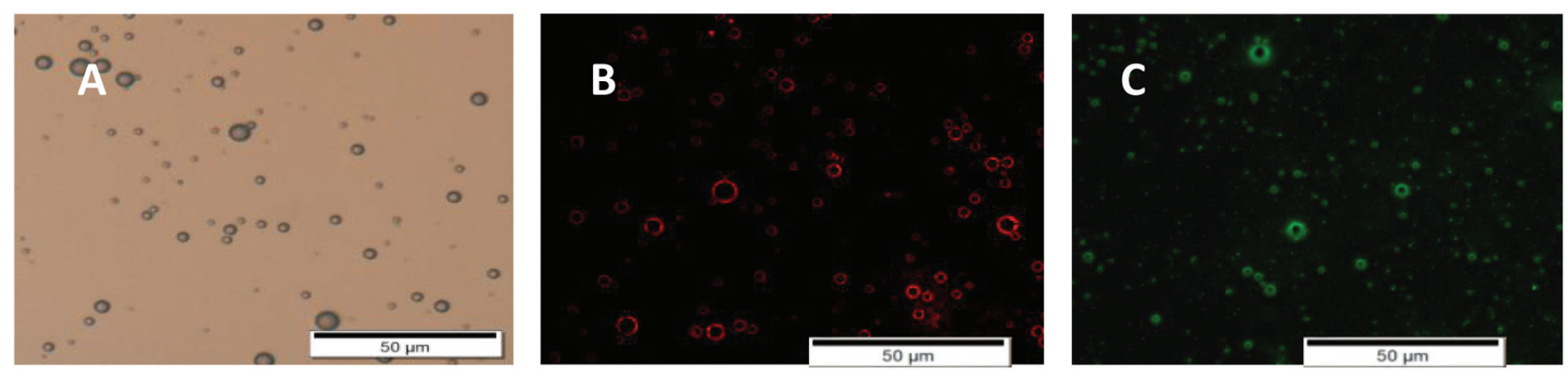

D
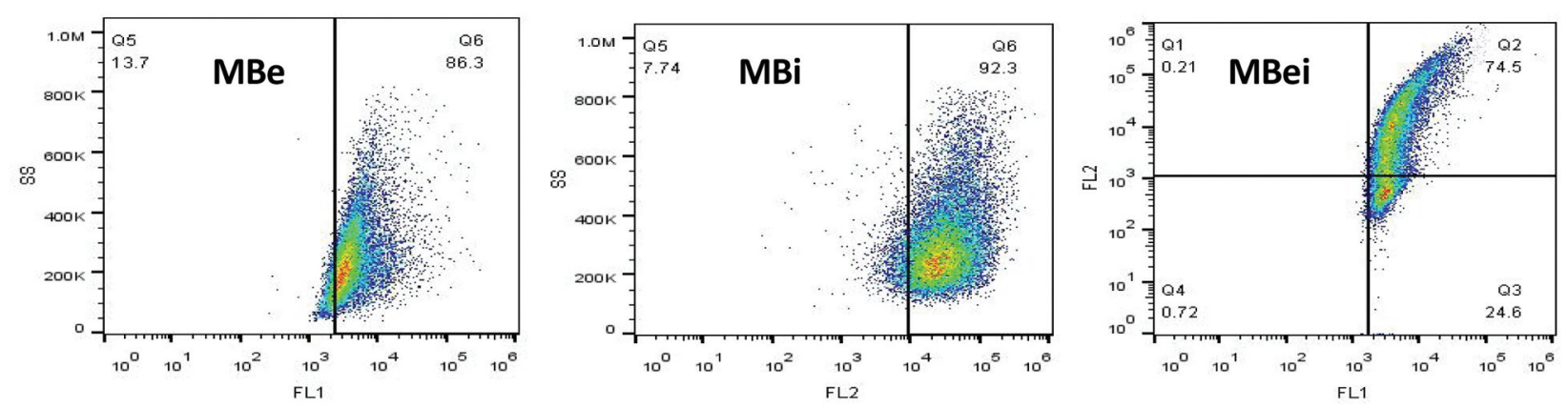

E

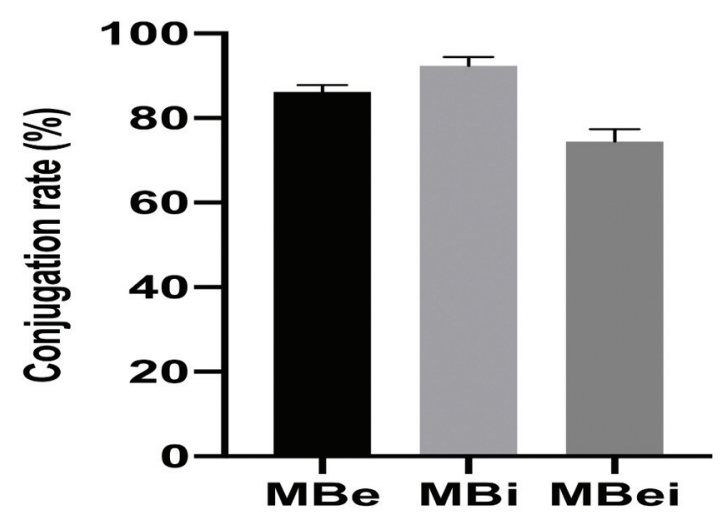

Figure 3. Representative images of ICAM-1- or Endostar-loaded microbubbles. A: Naked-MBs; B: ICAM-1-loaded MBs; C: Endostar-loaded MBs; D: Flow cytometric analysis of conjugated microbubbles; E: Conjugation rate of MBe (86.3\%), MBi (92.3\%) and MBei (74.5\%).

and flow cytometry. As shown in Figure 3A-C, fluorescentlylabeled ICAM-1 antibody and Endostar were visualized on the surface of the microbubbles by epifluorescence microscopy. Flow-cytometric analysis showed the conjugation rate of $\mathrm{MBe}$ (86.3\%), MBi (92.3\%) and MBei (74.5\%) (Figure 3D).

Adhesion of conjugated microbubbles to LPS-induced vein endothelial cells. To determine the targeting efficiency of conjugated MBs to LPS-induced HUVECs, the adhesion assay was performed. As shown in Figure 4, significantly more ICAM-1-labeled MBs were bonded to the LPS-induced HUVECs compared to the naked MBs (MBi: $p=0.035$; MBei: $p=0.032$ ).
Effects of targeted microbubbles on mRNA expression of CD31, ET-1, vWF, ERK and NF-kB in LPS-induced HUVECs. mRNA expression of the AS-related inflammatory markers in the LPS-induced HUVECs was significantly upregulated compared to the non-induced HUVECs $(p<0.05)$. As shown in Figure 5, the MBei treated group showed significantly downregulated mRNA expression of ERK compared to the LPS control $(p=0.046)$ and MBe treatment group $(p=0.009)$. No significant difference was found between the LPS control and the MBe treatment group. Both $\mathrm{MBe}$ and MBei treatment significantly down-regulated mRNA expression of $\mathrm{vWF}$ compared to the LPS control (MBe: $p=0.046$; MBei: $p=0.018$ ). No significant difference 


\section{A}
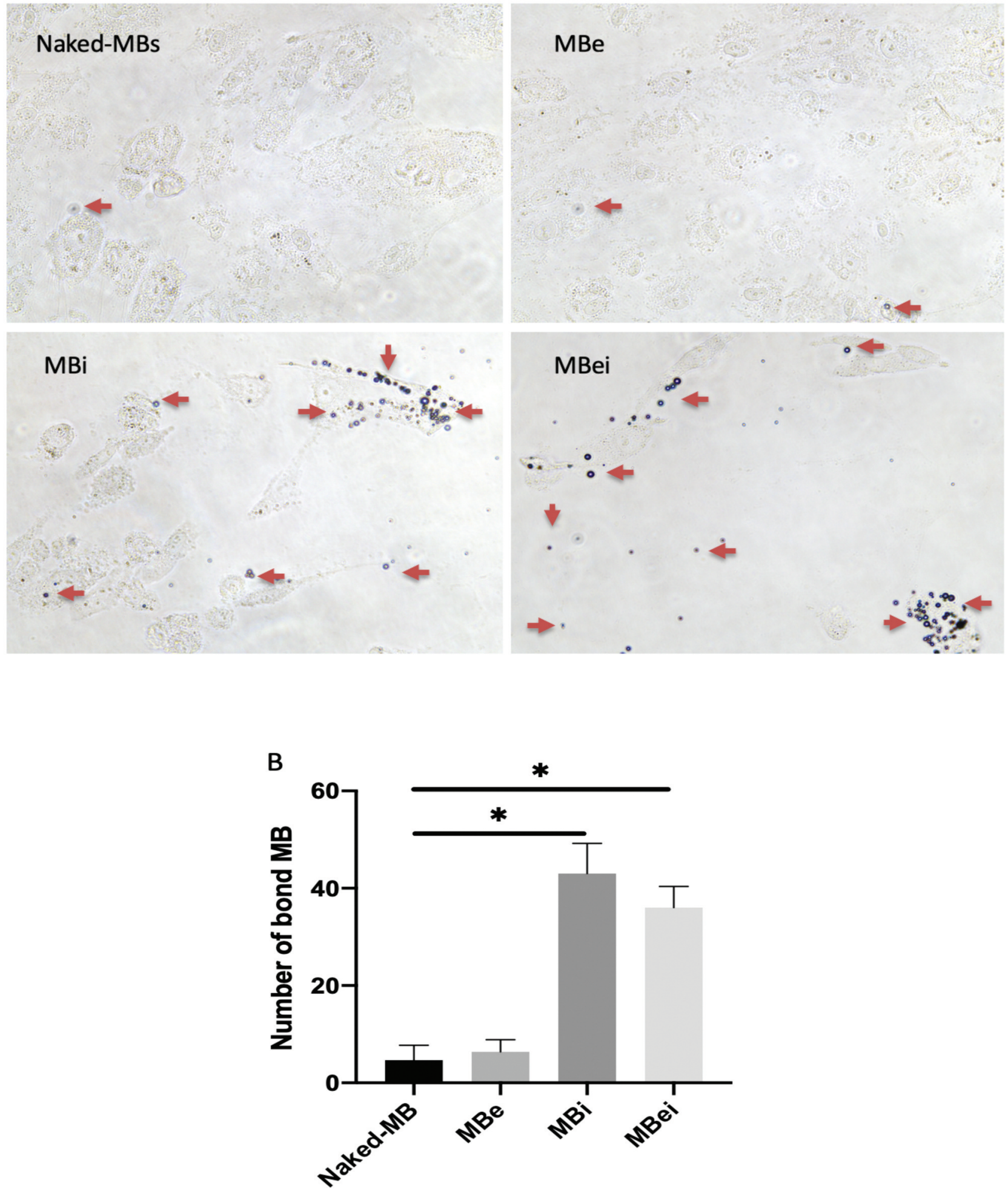

Figure 4. Adhesion of conjugated microbubbles to LPS-induced vein endothelial cells. A: Representative images of adhesion of conjugated microbubbles (red arrows) to LPS-induced HUVECs; B: Number of bonded MBs in each group. Significant differences between groups were calculated using one-way analysis of variance (ANOVA), followed by Tukey's correction. Data are presented as mean $\pm S D$. Statistical significance at $* p<0.05$. 


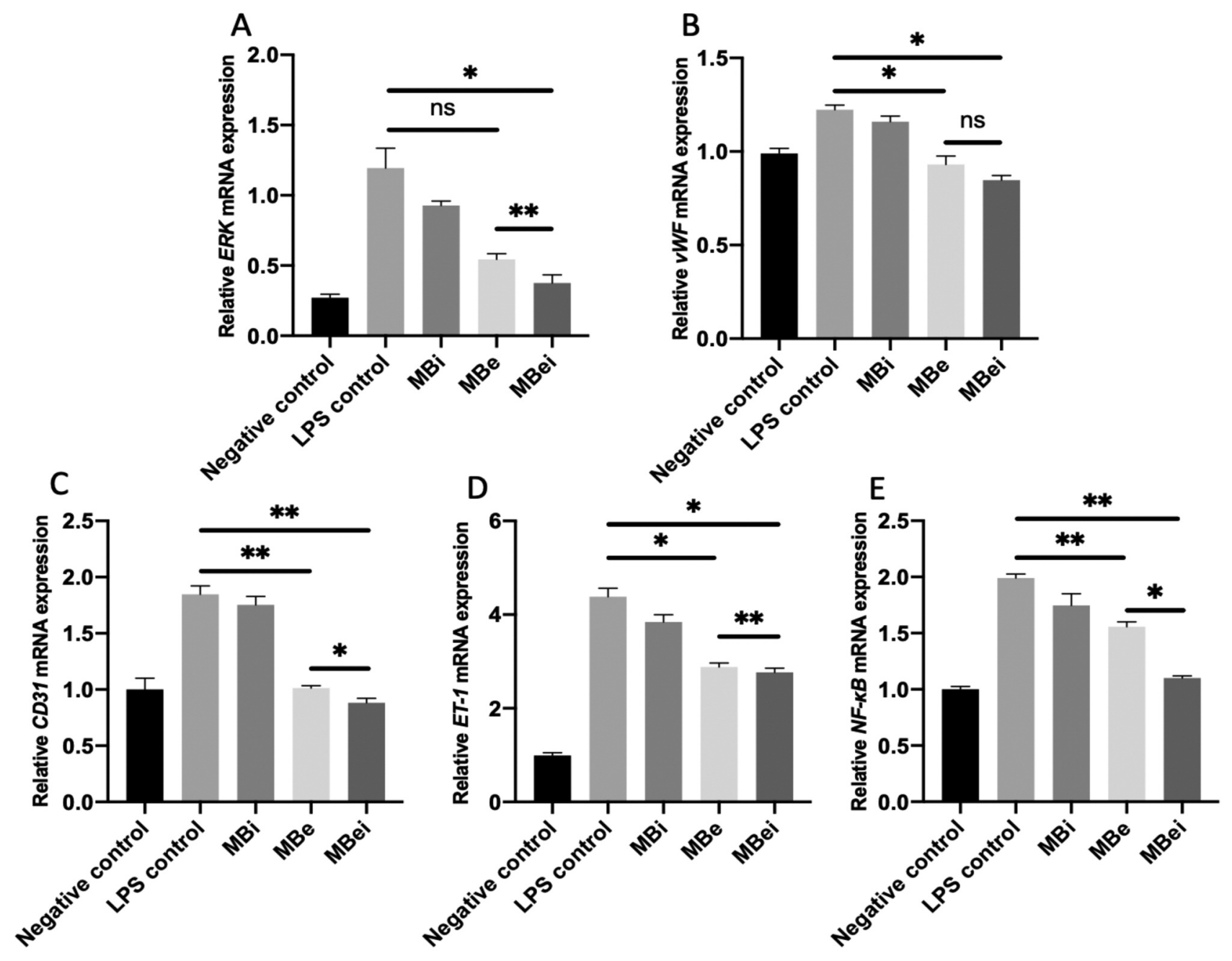

Figure 5. Effect of targeted microbubbles on mRNA expression of atherosclerosis-related inflammatory biomarkers in LPS-induced HUVECs. mRNA expression was determined using RT-PCR. A: ERK; B: vWF; C: CD31; D: ET-1; E: NF-kB. Significant differences between groups were calculated using one-way analysis of variance (ANOVA), followed by Tukey's correction. Data are presented as mean $\pm S D . * p<0.05$ and $* * p<0.01$.

was found between the MBe and the MBei treatment groups. Both MBe and MBei treatment significantly down-regulated mRNA expression of ET-1 (MBe: $p=0.012$; MBei: $p=0.011$ ), CD31 (MBe: $p=0.004$; MBei: $p=0.004$ ) and NF-kB (MBe: $p=0.007$; MBei: $p=0.003)$ compared to the LPS control. Furthermore, MBei showed significantly higher inhibitory efficacy compared to the MBe treatment group (ET-1: $p=0.001$; CD31: $p=0.046$; NF-kB: $p=0.021$ ).

Effects of targeted microbubbles on release of TF, TF-PI, ET$1, v W F$ and FVII $\alpha$ in LPS induced HUVECs. LPS induction significantly increased the release of the AS-related inflammatory cytokines $(p<0.05)$. After treatment with the MBs, the concentration of TF, TF-PI and vWF was significantly decreased in the MBei treatment group compared to the LPS control (TF: $p=0.033$, TF-PI: $p=0.019$ and vWF: $p=0.030)$ and MBe (TF: $p=0.012$, TF-PI: $p=0.046$ and vWF: $p=0.030)$ treatment groups, but no statistically significant differences were found between the LPS control and the $\mathrm{MBe}$ treatment group. FVII $\alpha$ was significantly decreased in both $\operatorname{MBe}(p=0.004)$ and MBei $(p=0.000)$ treatment groups compared to the LPS control, while MBei showed significantly higher inhibitory efficacy than the MBe treatment group $(p=0.033)$. ET-1 was significantly decreased in both $\mathrm{MBe}(p=0.035)$ and MBei $(p=0.014)$ treatment groups compared to the LPS control. No significant differences were found between the MBe and MBei groups ( $>0.05)$ (Figure 6). 

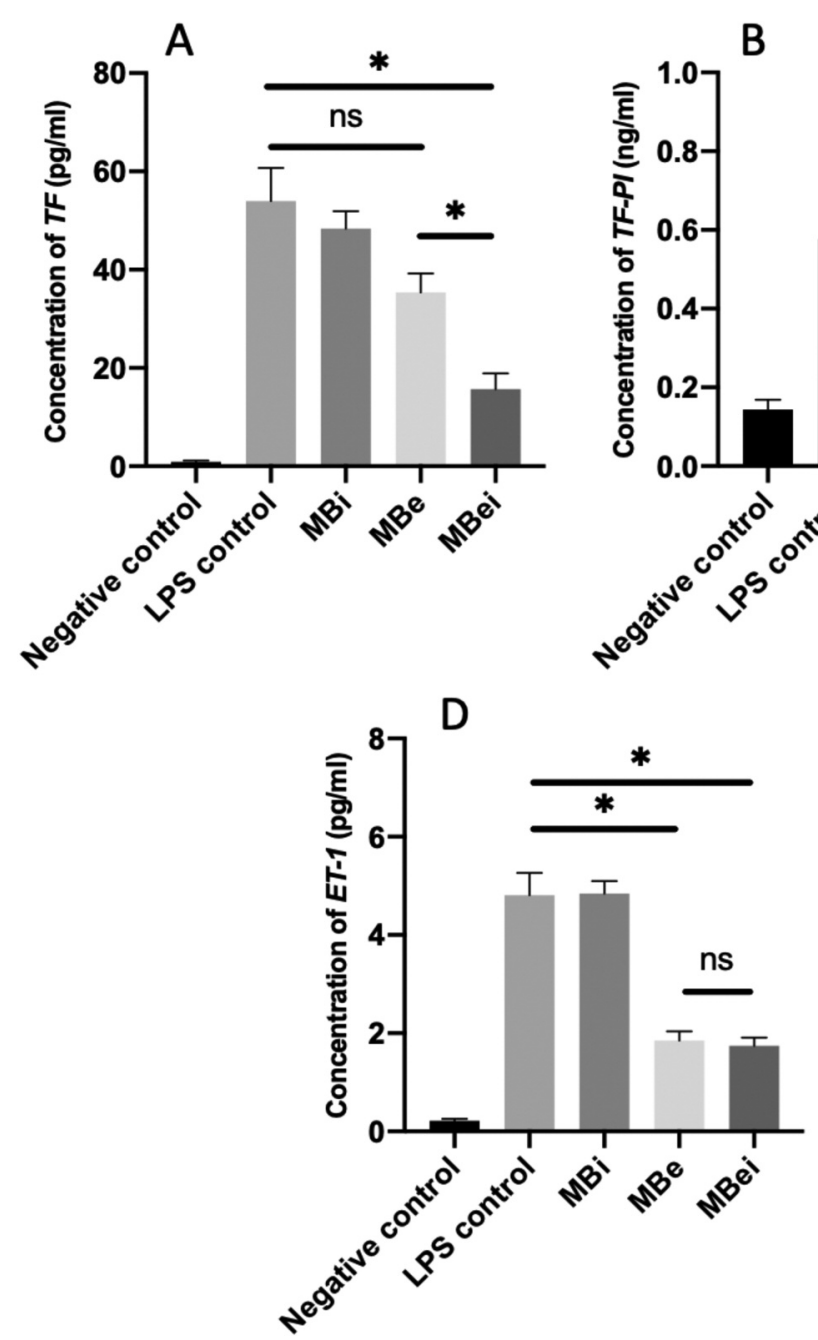

B

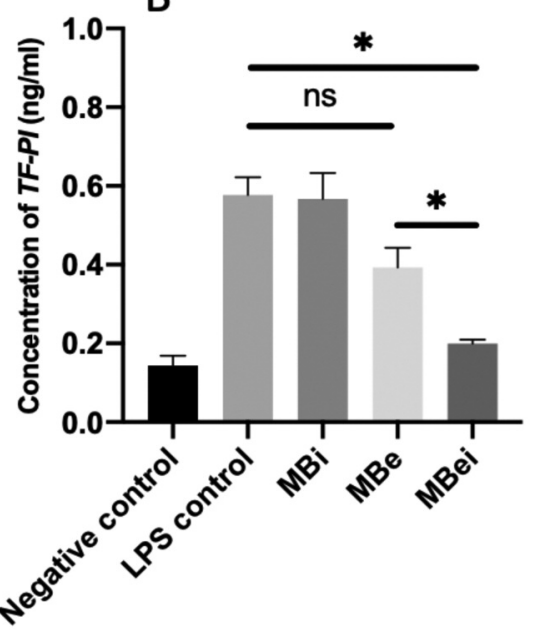

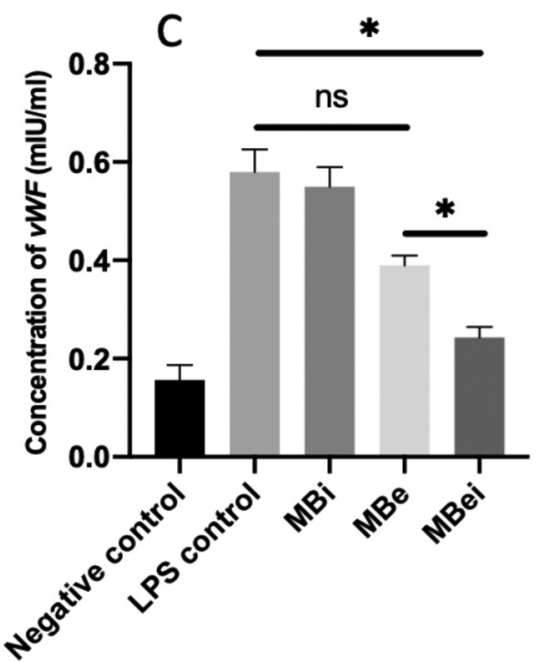

Figure 6. Effect of targeted microbubbles on release of atherosclerosis-related inflammatory cytokines in LPS-induced HUVECs. Cytokine levels were determined using ELISA. A: TF; B: TF-PI; C: vWF; D: ET-1; E: FVII . Significant differences between groups were calculated using oneway analysis of variance (ANOVA) followed by Tukey's correction. Data are presented as mean $\pm S D$. * $p<0.05$, **p<0.01 and $* * * * p<0.0001$.

\section{Discussion}

Inflammatory cells and cytokines were known to have an essential role in the development of atherosclerotic lesions (5) as well as accumulated plasma lipids (cholesterol and triglycerides) and lipoproteins (12-14). In hyperlipidemic plasma, oxidized-LDL (low density lipoprotein) binds with the endothelium and leads to a decrease of the NO concentration and endothelial activation $(15,16)$. This marks the onset of an inflammatory process, that further causes endothelial extravasation through the production of selectins and integrins such as P-selectin, E-selectin and ICAM-1 (17, 18). Following this, chemokines, such as interleukin (IL)-8, IL-6, angiotensin II and matrix-metalloproteinases (MMPs) are released at the inflammatory location and eventually lead to plaque formation (19-21).

We previously reported that treatment with UTMD targeting Endostar and ICAM-1 inhibited atherosclerotic plaques in animal models (6). Endostar is a modified version of recombinant human endostatin, which is a strong inhibitor of angiogenesis (22) and plaque neovascularization (23). ICAM-1 is an endothelial adhesion molecule that is upregulated in AS and is a target for UTMD therapy $(24,25)$. Villanueva et al. (26) reported that gas-filled microbubbles conjugated with anti-ICAM-1 antibody on their shell specifically bound to activated ECs overexpressing ICAM1, using a parallel-plate flow chamber. In the present study, we established a monolayer of activated HUVECs to mimic 
the inflammation of early-stage AS. The anti-ICAM-1Endostar-loaded MBs were perfused into a parallel-plate flow chamber. MBs were lysed by high acoustic pressure on the HUVECs and the Endostar was released directionally (27). The decreased expression of anti-angiogenesis and inflammatory cytokines in the treated HUVECs indicated that UTMD is an effective therapeutic for AS disease.

In conclusion, this is the first demonstration of antiinflammatory efficacy of targeted microbubbles on an earlystage AS model in vitro. In combination with our previous in vivo experiment (6), we believe that UTMD therapy has clinical potential for AS treatment.

\section{Conflicts of Interest}

None of the Authors have any conflicts of interest with regards to this study.

\section{Authors' Contributions}

JS designed the study; JS and YS analyzed the data and wrote the draft manuscript; YS and ZY participated in the study design; JS, $\mathrm{SP}$ and $\mathrm{HH}$ performed experiments; $\mathrm{ZY}$ and $\mathrm{RMH}$ revised the manuscript; HY supervised the study.

\section{References}

1 Libby P: Inflammation in atherosclerosis. Nature 420(6917): 868-874, 2002. PMID: 12490960. DOI: 10.1038/nature01323

2 Willerson JT and Ridker PM: Inflammation as a cardiovascular risk factor. Circulation 109(21 Suppl 1): II2-I10, 2004. PMID: 15173056. DOI: 10.1161/01.CIR.0000129535.04194.38

3 Szmitko PE, Wang CH, Weisel RD, de Almeida JR, Anderson TJ and Verma S: New markers of inflammation and endothelial cell activation: Part I. Circulation 108(16): 1917-1923, 2003. PMID: 14568885. DOI: 10.1161/01.CIR.0000089190.95415.9F

4 Hansson GK and Edfeldt K: Toll to be paid at the gateway to the vessel wall. Arterioscler Thromb Vasc Biol 25(6): 1085-1087, 2005. PMID: 15923538. DOI: 10.1161/01.ATV.0000168894. 43759.47

5 Pejkov H, Kedev S, Panov S, Srbinovska-Kostovska E and Lang I: Atherosclerosis of coronary blood vessels - local or systemic inflamation? Pril (Makedon Akad Nauk Umet Odd Med Nauki) 34(3): 5-11, 2013. PMID: 24566010.

6 Yuan H, Hu H, Sun J, Shi M, Yu H, Li C, Sun YU, Yang Z and Hoffman RM: Ultrasound microbubble delivery targeting intraplaque neovascularization inhibits atherosclerotic plaque in an APOE-deficient mouse model. In Vivo 32(5): 1025-1032, 2018. PMID: 30150423 . DOI: 10.21873 /invivo. 11342

7 Levesque MJ and Nerem RM: The elongation and orientation of cultured endothelial cells in response to shear stress. J Biomech Eng 107(4): 341-347, 1985. PMID: 4079361. DOI: 10.1115/1.3138567

8 Wang YX, Xiang C, Liu B, Zhu Y, Luan Y, Liu ST and Qin KR: A multi-component parallel-plate flow chamber system for studying the effect of exercise-induced wall shear stress on endothelial cells. Biomed Eng Online 15(Suppl 2): 154, 2016. PMID: 28155716. DOI: 10.1186/s12938-016-0273-Z
9 Li X, Chen W, Li P, Wei J, Cheng Y, Liu P, Yan Q, Xu X, Cui Y, Gu Z, Simoncini T and Fu X: Follicular stimulating hormone accelerates atherogenesis by increasing endothelial VCAM-1 expression. Theranostics 7(19): 4671-4688, 2017. PMID: 29187895. DOI: $10.7150 /$ thno. 21216

10 Lawrence MB, McIntire LV and Eskin SG: Effect of flow on polymorphonuclear leukocyte/endothelial cell adhesion. Blood 70(5): 1284-1290, 1987. PMID: 3663936.

11 Patton JT, Menter DG, Benson DM, Nicolson GL and McIntire LV: Computerized analysis of tumor cells flowing in a parallel plate chamber to determine their adhesion stabilization lag time. Cell Motil Cytoskeleton 26(1): 88-98, 1993. PMID: 8106174. DOI: $10.1002 / \mathrm{cm} .970260109$

12 Kannel WB, Castelli WP and Gordon T: Cholesterol in the prediction of atherosclerotic disease. New perspectives based on the Framingham study. Ann Intern Med 90(1): 85-91, 1979. PMID: 217290. DOI: 10.7326/0003-4819-90-1-85

13 Martin NA, Hadley MN, Spetzler RF and Carter LP: Management of asymptomatic carotid atherosclerosis. Neurosurgery 18(4): 505-513, 1986. PMID: 3517677. DOI: 10.1227/00006123-198604000-00026

14 Lloyd-Jones DM, O’Donnell CJ, D’Agostino RB, Massaro J, Silbershatz $\mathrm{H}$ and Wilson PW: Applicability of cholesterollowering primary prevention trials to a general population: the framingham heart study. Arch Intern Med 161(7): 949-954, 2001. PMID: 11295957. DOI: 10.1001/archinte.161.7.949

15 Alderman CJ, Bunyard PR, Chain BM, Foreman JC, Leake DS and Katz DR: Effects of oxidised low density lipoprotein on dendritic cells: a possible immunoregulatory component of the atherogenic micro-environment? Cardiovasc Res 55(4): 806-819, 2002. PMID: 12176130. DOI: 10.1016/s0008-6363(02)00447-9

16 Fontana L, Giagulli C, Cominacini L, Pasini AF, Minuz P, Lechi A, Sala A and Laudanna C: Beta2 integrin-dependent neutrophil adhesion induced by minimally modified low-density lipoproteins is mainly mediated by F2-isoprostanes. Circulation 106(19): 2434-2441, 2002. PMID: 12417539. DOI: 10.1161/ 01.cir.0000037223.92135.38

17 Dong ZM, Brown AA and Wagner DD: Prominent role of Pselectin in the development of advanced atherosclerosis in ApoE-deficient mice. Circulation 101(19): 2290-2295, 2000. PMID: 10811597. DOI: 10.1161/01.cir.101.19.2290

18 Piqueras L, Kubes P, Alvarez A, O’Connor E, Issekutz AC, Esplugues JV and Sanz MJ: Angiotensin II induces leukocyteendothelial cell interactions in vivo via $\mathrm{AT}(1)$ and $\mathrm{AT}(2)$ receptormediated P-selectin upregulation. Circulation 102(17): 2118-2123, 2000. PMID: 11044430. DOI: 10.1161/01.cir.102.17.2118

19 Wang N, Tabas I, Winchester R, Ravalli S, Rabbani LE and Tall A: Interleukin 8 is induced by cholesterol loading of macrophages and expressed by macrophage foam cells in human atheroma. J Biol Chem 271(15): 8837-8842, 1996. PMID: 8621523. DOI: $10.1074 /$ jbc.271.15.8837

20 Libby P and Schönbeck U: Drilling for oxygen: angiogenesis involves proteolysis of the extracellular matrix. Circ Res 89(3): 195-197, 2001. PMID: 11485968.

21 Rajavashisth TB, Liao JK, Galis ZS, Tripathi S, Laufs U, Tripathi J, Chai NN, Xu XP, Jovinge S, Shah PK and Libby P: Inflammatory cytokines and oxidized low density lipoproteins increase endothelial cell expression of membrane type 1-matrix metalloproteinase. J Biol Chem 274(17): 11924-11929, 1999. PMID: 10207013. DOI: 10.1074/jbc.274.17.11924 
22 Ling Y, Yang Y, Lu N, You QD, Wang S, Gao Y, Chen Y and Guo QL: Endostar, a novel recombinant human endostatin, exerts antiangiogenic effect via blocking VEGF-induced tyrosine phosphorylation of KDR/Flk-1 of endothelial cells. Biochem Biophys Res Commun 361(1): 79-84, 2007. PMID: 17644065. DOI: $10.1016 /$ j.bbrc.2007.06.155

23 Mao W, Kong J, Dai J, Huang ZQ, Wang DZ, Ni GB and Chen ML: Evaluation of recombinant endostatin in the treatment of atherosclerotic plaques and neovascularization in rabbits. $\mathrm{J}$ Zhejiang Univ Sci B 11(8): 599-607, 2010. PMID: 20669350. DOI: 10.1631 jzus.B1001011

24 Galkina $\mathrm{E}$ and Ley $\mathrm{K}$ : Vascular adhesion molecules in atherosclerosis. Arterioscler Thromb Vasc Biol 27(11): 22922301, 2007. PMID: 17673705. DOI: 10.1161/ATVBAHA. 107.149179

25 Bourdillon MC, Poston RN, Covacho C, Chignier E, Bricca G and McGregor JL: ICAM-1 deficiency reduces atherosclerotic lesions in double-knockout mice (ApoE(-/-)/ICAM-1(-/-)) fed a fat or a chow diet. Arterioscler Thromb Vasc Biol 20(12): 26302635, 2000. PMID: 11116064. DOI: 10.1161/01 atv.20.12.2630
26 Villanueva FS, Jankowski RJ, Klibanov S, Pina ML, Alber SM, Watkins SC, Brandenburger GH and Wagner WR: Microbubbles targeted to intercellular adhesion molecule-1 bind to activated coronary artery endothelial cells. Circulation 98(1): 1-5, 1998. PMID: 9665051. DOI: 10.1161/01.cir.98.1.1

27 Zhang C, Huang P, Zhang Y, Chen J, Shentu W, Sun Y, Yang Z and Chen S: Anti-tumor efficacy of ultrasonic cavitation is potentiated by concurrent delivery of anti-angiogenic drug in colon cancer. Cancer Lett 347(1): 105-113, 2014. PMID: 24530229. DOI: $10.1016 /$ j.canlet.2014.01.022

Received April 23, 2021

Revised July 10, 2021

Accepted August 20, 2021 